\title{
FORÇA MUSCULAR DE MEMBROS INFERIORES E CAPACIDADE FUNCIONAL DE IDOSAS PRATICANTES DE PROGRAMA DE EXERCÍCIO FÍSICO SUPERVISIONADO
}

Juliana Rosini da Silva ${ }^{1}$; Tabata de Paula Facioli ${ }^{1}$, Claudia Regina Sgobbi de Faria ${ }^{1}$; Regina Celi Trindade Camargo ${ }^{1}$.

1. Departamento de Fisioterapia - Faculdade de Ciências e Tecnologia FCT/UNESP - Presidente Prudente juliana rosini@yahoo.com.br

\section{RESUMO}

Sabe-se que um dos mais evidentes fatores que restringem a vida do idoso é a incapacidade funcional e o declínio da força. Por meio de programas de exercícios supervisionados, pode-se reverter este quadro. Avaliar e correlacionar a capacidade funcional e a força muscular de idosas ativas. Participaram do estudo 31 indivíduos do sexo feminino, integrantes de Programa de Fisioterapia Preventiva. Para a pesquisa foram aplicados os testes TC6 e Dinamometria pré e após seis meses de intervenção. A estatística foi expressa por valores de média e desvio-padrão (DP). 0 nível de significância utilizado foi de $p<0.05$. Houve diferença significativa quando comparados os períodos pré e pós-intervenção na força no movimento de extensão de joelho. O protocolo de exercícios foi capaz de promover melhoras na capacidade funcional, no equilíbrio, flexibilidade e força das participantes, mostrando ser eficaz na redução dos efeitos deletérios advindos do envelhecimento.

Palavras-chave: idoso, programa de prevenção, capacidade funcional, força .

\section{INTRODUÇÃO}

Com o avanço da medicina e melhora da qualidade de vida da população, a expectativa de vida do brasileiro que em 2008 era de 72,7 anos pode passar a ser de 81,29 anos em 2050 ${ }^{1}$. Apesar da expectativa de vida e de sua qualidade estarem em ascensão, inúmeros são os efeitos deletérios advindos da terceira idade. São observados no decorrer do processo de envelhecimento significativos déficits na capacidade funcional, principalmente em seu fator força muscular ${ }^{2,3,4}$.

De acordo com diversos estudos, a força muscular máxima é obtida até a 3a década de vida, e mantida até os 50 anos de idade, onde começa seu declínio. Até os 70 anos há uma perda de $15 \%$ a cada década vivida, e após essa idade esse fenômeno aumenta em 30\% no mesmo espaço de tempo. A perda de massa muscular, seja por atrofia ou diminuição de fibras musculares, é a principal causa na redução da força muscular ${ }^{5}$. A redução da massa muscular pode estar associada tanto ao metabolismo quanto ao nível de atividade física praticada pelo idoso ${ }^{6,7}$.

Há evidencias de que a participação em programas de exercícios regulares, voltados para o desenvolvimento da capacidade funcional, seja um efetivo caminho para reverter e/ou prevenir os inúmeros declínios associados ao envelhecimento. A Organização Mundial da Saúde (OMS) 
considera que países em desenvolvimento como o Brasil, seus idosos podem perder até $5 \%$ da sua capacidade física a cada 10 anos, podendo recuperar $10 \%$ desta perda, por meio de atividades físicas adequadas ${ }^{8,9}$.

Sendo assim o presente estudo tem como interesse avaliar e correlacionar a capacidade funcional e a força muscular de membros inferiores de idosos participantes de um programa de prevenção aos efeitos deletérios da terceira idade.

\section{OBJETIVOS}

Avaliar e correlacionar capacidade funcional e força muscular de idosas participantes de um programa de exercícios supervisionados de fisioterapia preventiva, no período de seis meses de intervenção.

\section{METODOLOGIA}

Trata-se de um estudo quantitativo com amostra por conveniência, composta de idosos do sexo feminino, participantes do programa de fisioterapia para a terceira idade do Setor de Gerontologia do Núcleo Morumbi da Faculdade de Ciências e Tecnologia - FCT/UNESP.

Como critérios de inclusão foram consideradas idosas com idade igual ou superior a 60 anos, participantes do programa de fisioterapia e prevenção aos efeitos deletérios da terceira idade da FCT/UNESP, com frequência de duas vezes semanais. Cada sessão tem duração de 60 minutos, e composta por exercícios de alongamento, aquecimento, fortalecimento muscular, coordenação motora, equilíbrio, dinâmicas lúdicas e relaxamento, sendo aferidos parâmetros vitais no início e final de cada sessão. As avaliações foram realizadas no Setor de Gerontologia do Centro de Estudos e Atendimentos em Fisioterapia e Reabilitação (CEAFIR) da Faculdade de Ciências e Tecnologia - FCT/UNESP, onde ocorreram as atividades propostas do programa. $O$ estudo teve duração de seis meses, onde as participantes foram avaliadas pré e pós o período de intervenção.

Para avaliação da funcionalidade foi utilizado o teste de caminhada de seis minutos (TC6), por ser um teste simples, de fácil aplicação e bem tolerado por idosos, ao permitir livre escolha da velocidade de caminhada ${ }^{10}$. O teste foi realizado de acordo com as diretrizes da American Thoracic Society $(2002)^{11}$, duas vezes no mesmo dia com intervalo de 20 minutos entre elas. Os dados vitais foram coletados e registrados no início e ao final do teste. 
A mensuração da força dos membros inferiores, foi realizada unilateralmente (membro dominante), por meio de dinamômetro digital da marca Force Gauge ${ }^{\circledR}$, modelo FG - $100 \mathrm{~kg}$. Os resultados foram expressos em Newtons (N). Cada participante foi orientada a executar o movimento (extensão e flexão de joelho), contra a resistência de uma faixa acoplada ao dinamômetro. A participante realizou, portanto contração isométrica voluntária máxima (CIVM) durante 6 segundos, seguida de relaxamento do membro. A medida foi repetida três vezes, com um intervalo de 1 minuto entre elas, e o maior valor registrado.

A estatística descritiva foi composta por valores de média e desvio-padrão (DP). As comparações entre o momento pré e pós foram efetuadas pelo teste $t$ de Student para dados pareados, a correlação foi realizada por meio do coeficiente de Pearson, de acordo com a normalidade dos dados. O nível de significância utilizado foi de $p<0.05$. As análises foram efetuadas no software BioEstat versão 5.0. O presente estudo contou com a aprovação do Comitê de Ética em Pesquisa desta Instituição Número (CAAE no 02798312.8.0000.5402)

\section{RESULTADOS}

A amostra do estudo foi composta por 31 indivíduos do sexo feminino, com idade entre 60 a 88 anos $(73,8 \pm 7,1$ anos).

Na comparação da variável capacidade funcional, não houve diferença significativa no TC6 $(P=0.07)$, apesar do aumento da mesma no período analisado, como representado na tabela 1. Para análise da força muscular de membros inferiores pré e pós intervenção, houve diferença significativa no movimento de extensão de joelho $(P=0.04)$, como mostra a tabela 2.

De acordo com os dados analisados, houve correlação positiva entre os valores de capacidade funcional e força muscular de membros inferiores ( $p=0,005$ e $r=0,51$ para extensão de joelho, e $p=0,01$ e $r=0,44$ para flexão de joelho).

TABELA 1. Efeitos do programa de exercícios sobre a capacidade nos períodos pré e pós seis meses de tratamento.

\section{Capacidade Funcional $(n=31)$}

\begin{tabular}{cccc}
\hline & Pré & Pós & P-valor \\
\hline TC6 & $471,6 \pm 81,8$ & $487,5 \pm 92,2$ & 0.07 \\
\hline \hline
\end{tabular}


Dados apresentados em média e desvio padrão.

Abreviações: $\mathrm{cm}=$ centímetros.

*Considerado estatisticamente significante $\mathrm{P}$ valor $<0.05$.

TABELA 2. Efeitos do programa de exercícios sobre a força muscular de membros inferiores nos períodos pré e pós seis meses de tratamento.

\section{Força $(n=31)$}

\begin{tabular}{cccc}
\hline & Pré & Pós & P-valor \\
Extensão de joelho $(\mathbf{N})$ & $150.8 \pm 58.8$ & $171.0 \pm 51.7$ & $0.04^{*}$ \\
\hline Flexão de joelho $(\mathrm{N})$ & $54.2 \pm 20.5$ & $60.7 \pm 18.6$ & 0.2 \\
\hline \hline
\end{tabular}

Dados apresentados em média e desvio padrão.

Abreviações: $\mathrm{cm}=$ centímetros.

*Considerado estatisticamente significante $\mathrm{P}$ valor $<0.05$.

\section{DISCUSSÃO}

Após o período de seis meses de aplicação de um protocolo de exercícios, não houve declínio em nenhuma das variáveis analisadas. No presente estudo não foi encontrada diferença significante entre os valores referentes à capacidade funcional, observada por meio do TC6, mas pode ser observada uma melhora clínica significante após o período de intervenção. O TC6 mostrou-se um eficiente indicador da capacidade funcional, sendo um método de fácil aplicação, seguro e de baixo custo ${ }^{11}$.

A mobilidade é considerada a variável fundamental da capacidade funcional, sendo determinada pela velocidade do andar, atividade esta que é considerada essencial para a realização das atividades de vida diária de qualquer individuo, mas que são afetadas durante o processo de envelhecimento tornando-se lenta ${ }^{12,13}$. Porém, a amostra deste estudo não apresentou déficit de mobilidade quanto ao nível de atividade física no período de seis meses, independente da idade cronológica das participantes quanto ao envelhecimento individual.

Em relação aos valores obtidos nos testes de força, foi demonstrada a efetividade do programa proposto, uma vez que houve aumento da força muscular de todas as participantes em ambos os movimentos analisados, mesmo o programa de exercícios ocorrendo duas vezes semanais. Porter et al. ${ }^{14}$ e Carvalho et al. ${ }^{15}$ ao compararem a influência da frequência semanal no 
incremento da força muscular, demonstrou que a frequência de 2 dias por semana seria suficiente para a melhora desta variável em idosas.

Os efeitos do envelhecimento levam ao declínio da força muscular, resultado da redução da velocidade de contração das fibras musculares bem como a diminuição do número das mesmas, podendo afetar a capacidade funcional, entretanto este fato não foi encontrado no presente estudo, durante o período analisado, provavelmente dada a participação efetiva no programa de exercícios e pelo nível de envolvimento com atividades de vida diária das participantes $^{16}$.

O aumento da capacidade funcional e força de membros inferiores estão diretamente relacionados, uma vez que a força oferece suporte para a realização das atividades que envolvem a mobilidade e a habilidade de caminhar, como visto neste estudo.

\section{CONCLUSÃO}

O programa de exercícios supervisionados de fisioterapia preventiva pode oferecer inúmeros benefícios, resultando em independência pessoal e melhora da força muscular para quem adere a esta prática. Sendo assim, o programa proposto, é um exemplo de prática de exercício físico de baixo custo, que oferece às participantes benefícios capazes de minimizarem efeitos deletérios do processo de envelhecimento.

\section{REFERÊNCIAS}

1. Instituto Brasileiro de Geografia e Estatística (IBGE) 2009. Estimativas populacionais. Rio de Janeiro, 2009.

2. Deschenes MR. Effects of aging on muscle fibre type and size. Sports Med 2004;34(12):809-24. http://dx.doi.org/10.2165/00007256-200434120-00002

3. Holland GJ, Tanaka K, Shigematsu R, Nakagaichi M. Flexibility and physical functions of older adults: A Review. J Aging Phys Act 2002;10(2):169-206.

4. Fatouros IG, Kambas A, Katrabasas I, Leontsini D, Chatzinikolaou A, Jamurtas AZ, et al. Resistance training and detraining effects on flexibility performance in the elderly are intensitydependent. J Strength Cond Res 2006; 20(3):634-642.

5. Matsudo SM., Marin RV. , Ferreira MT., Araújo TL. Estudo longitudinal- tracking de 4 anos - da aptidão física de mulheres da maioridade fisicamente ativas. Revista Brasileira de Ciência e Movimento, Brasília, v.12, n.3, p.47-52, 2004.

6. Nair KS. Aging muscle. Am J Clin Nutr 2005;81(5):953-63. 
7. Hunter GR, McCarthy JP. Bamman MM. Effects of resistance training on older adults. Sports Med 2004;34(5):329-48. http://dx.doi.org/10.2165/00007256-200434050-00005

8. American College Of Sport Medicine.Exercício e Envelhecimento. Rev Virtual EF Artigos. 2004; 2(5). Disponível em: <http://efartigos.atspace.org/otemas/artigo29.html>. Acesso em: 15 de outubro de 2009.

9. Freitas CMSM, Santiago MS, Viana AT, Leão AC, Freyre C. Aspectos motivacionais que influenciam a adesão e manutenção de idosos a programa de exercícios físicos. Rev Bras Cineantropom Desempenho Hum. 2007; 9(1): 92-100.

10. Brooks D, Solway S, Gibbons WJ. ATS statement: guidelines for the six-minute walk test. Am J Respir Crit Care Med. 2002;166 (1):111-7. http://dx.doi.org/10.1164/ajrccm.166.1.at1102

11. ATS statement: guidelines for the six-minute walk test. ATS Committee on Proficiency Standards for Clinical Pulmonary Function Laboratories. Am J Respir Crit Care Med 2002;166(1):111-117. http://dx.doi.org/10.1164/ajrccm.166.1.at1102

12. La Croix AZ, Guralnik JM, Berkman LF, Wallace RB, Satterfield S. Maintaining mobility in late life . II. Smoking, alcohol consumption, physical activity, and body mass index. Am J Epidemiol 1993; 137(8):858-869.

13. Mc Ginn AP, Kaplan RC, Verghese J, Rosenbaum DM, Psaty BM, Baird AE, Lynch JKA, Wolf AP, Kooperberg C, Larson JC, Wassertheil-Smoller S. Walking speed and risk of incident ischemic stroke $\begin{array}{llll}\text { among } & \text { postmenopausal }\end{array}$ http://dx.doi.org/10.1161/STROKEAHA.107.500850

14. Porter MM, Nelson ME, Fiatarone SMA, Layne JE, Morganti CM, Trice I, et al. Effects of longterm resistance training and detraining on strength and physical activity in older women. J Aging Phys Activity 2002;10:260-270.

15. Carvalho J, Oliveira J, Magalhães J, Ascensão A, Mota J, Soares JMC. Força muscular em idosos II - Efeito de um programa complementar de treino na força muscular de idosos de ambos os sexos. Rev Port Ciênc Des 2004;4:58-65.

16. Matsudo SM, Matsudo VK, Barros TL. Impacto do envelhecimento nas variáveis antropométricas, neuromotoras e metabólicas da aptidão física. Ver Bras Ciência e Movimento 2000; 8(4): 21-32. 